\title{
José de Acosta y la antropología renacentista. Reflexión sobre la antropología y etnografía de los humanistas
}

\author{
IRIS GAREIS
}

\section{RESUMEN}

La obra del padre José de Acosta tuvo enorme influencia sobre sus contemporáneos y también sobre las siguientes generaciones de autores de diversas disciplinas. El artículo explora de qué manera los escritos de Acosta superaron los textos etnográficos anteriores de los humanistas renacentistas y analiza las teorías más importantes presentes en la obra del padre jesuita.

Palab Ras Clave: Etnografia y antropología renacentistas, teoría política, historia universal, bistoria de la ciencia, evangelización 


\begin{abstract}
José de Acosta's work exerted enormous influence on his contemporaries as well as on authors of future generations of diverse disciplines. The article explores the ways in which Acosta's writings excelled former ethnographic texts of Renaissance humanists and discusses the most important theories developed by the Jesuit father.
\end{abstract}

KeYwords: Renaissance ethnography and anthropology, political theory, universal history, history of science, evangelization

«Assi que aunque el mundo nueuo ya no es nueuo sino viejo, segun ay mucho dicho, y escripto del, toda via me parece que en alguna manera se podra tener esta Historia por nueua, por ser juntamente Historia y en parte Philosophia [...]»

(Acosta 1590: 10).

\title{
INTROducción: José De Acosta y LA TRADICIÓN HUMANISTA
}

El PRIMER viAje de Cristóbal Colón en 1492 cambió por completo y para siempre la imagen que los europeos hasta entonces habían tenido del mundo. Si bien los primeros navegantes no se dieron cuenta de inmediato que habían descubierto no solamente algunas «islas nuevas», sino todo un continente del cual hasta aquel entonces no habían tenido la menor noticia, no tardaron en avanzar explorando el mar de las Antillas hasta llegar a tierra firme. Este «Nuevo Mundo» que pronto se llamaría «América» despertó desde el primer momento la curiosidad de los humanistas. ¿Qué podría haber sido más apto en la época del Renacimiento, caracterizada por el afán de renovación de la herencia del mundo antiguo, que el hallazgo de nuevas tierras con sus habitantes exóticos, los cuales 
parecían tener semejanzas con los pueblos mencionados por los autores de la Antigüedad? Por consiguiente, los eruditos humanistas al poco rato de los primeros descubrimientos empezaron a producir un cuerpo de textos etnográficos describiendo el nuevo continente y sus pueblos. En la segunda mitad del siglo XVI las así llamadas «Americana», es decir libros sobre América, ya habían alcanzado cifras muy elevadas de ejemplares publicados. ${ }^{1}$

Las obras del jesuita José de Acosta publicadas en las dos últimas décadas del siglo XVI, por consiguiente, pertenecen a esta segunda fase de la etnografía y antropología americanas. Al iniciar sus dos obras magistrales, nuestro autor, por lo tanto, ya contaba con una base de datos etnográficos y una imagen elaborada de las Américas ofrecidas en gran cantidad de publicaciones. Sin embargo, como veremos en adelante, Acosta no se contentó con compilar informaciones etnográficas sobre las culturas americanas. Más bien trataba de dar respuestas a interrogantes típicamente antropológicas. En su primer libro (De Procuranda Indorum Salute, publicado en 1588) intentaba explicar los paralelos etnográficos entre las culturas andinas y europeas, los que había encontrado en el campo de la religión. Luego, en su segundo libro (Historia natural y moral de las Indias que publicó dos años después) enfocó, entre otros problemas, la diversidad de los pueblos americanos y el porqué la conquista y misión españolas fueron más exitosas con unos que con otros. Es decir que, en adición a nuevos datos etnográficos que había reunido durante su estadía en el Perú y en México, adelantaba hipótesis antropológicas como resultado de la comparación de diversas culturas americanas. Además de aquellas preguntas tocantes a la antropología, también discutía problemáticas pertenecientes a la complexión natural del continente americano y su lugar en la historia universal.

En lo siguiente nos ocuparemos, en primer lugar, de los rasgos característicos de la etnografía de los humanistas del Renacimien-

1 Agradezco a la Dra. María del Pilar Mejía Quiroga sus comentarios y sugerencias. 
to para entender sobre qué base José de Acosta elaboró su visión antropológica de los pueblos americanos y en qué medida esta se distinguía de las obras etnográficas anteriores. Luego, revisamos las obras de Acosta con respecto a las informaciones etnográficas y las interpretaciones antropológicas e históricas ofrecidas, para finalmente comprobar que con todo derecho su traductor al francés le otorgó el epíteto «el Heródoto o el Plinio de este mundo nuevamente descubierto». ${ }^{2}$ Por último, nos proponemos señalar la recepción y la influencia de sus obras en los escritos de autores posteriores.

\section{El «Nuevo Mundo» EN LA ETNOGRAfía RENACENTista}

Si bien los humanistas produjeron textos etnográficos, en su época no se conocía todavía el término «etnografía». Por lo tanto, al hablar de la «etnografía» renacentista recurrimos en cierto modo a una noción anacrónica, puesto que este neologismo fue recién creado en la segunda mitad del siglo XVIII. La creación de la palabra compuesta de los vocablos griegos «éthnos» con el significado «pueblo» o «nación»y del verbo «gráphein» que significa «escribir» se debe a dos eruditos de la universidad alemana de Göttingen. En 1772, August Ludwig Schlözer menciona por primera vez el término neológico y, en 1775, Johann Christoph Gatterer, profesor titular de historia, declara que la «etnografía» forma una de las cuatro secciones de la descripción del mundo (Gareis 2014: 358). En cierto modo esta definición refleja el afán de las grandes expediciones de la segunda mitad del siglo XVIII y de principios del siglo XIX de coleccionar una documentación exacta, detallada y completa sobre las regiones visitadas. Esto significa que la etnografía en esta época

2 La traducción de la cita original está tomada de Del Pino (2009: 112). Con respecto a Robert Regnault, traductor francés de la Historia de Acosta, véase Courcelles (2003: 311-313). 
ya no ocupaba un lugar principal del interés científico, sino tan sólo aportaba datos al cuadro general de la historia natural en conjunto con otras disciplinas, como por ejemplo la botánica, zoología, mineralogía, etc. (Gareis 2006: 594). Como veremos en adelante, esta visión de la etnografía como una parte de la descripción del mundo y, por lo tanto, de la antropología como la correspondiente parte de la explicación del mundo, también la encontramos en la Historia natural y moral de Acosta.

Al referirnos a la etnografía de la Antigüedad y de la Edad Moderna, entendemos por este término una descripción de una cultura, etnia o sociedad, en general diferente de aquella del etnógrafo y de sus lectores. ${ }^{3}$ Tanto la etnografía renacentista, como la antigua, se basaban en observaciones de los propios autores, pero también en conocimientos transmitidos en textos o en tradiciones orales y descripciones de viajeros o conquistadores. Schlözer y Gatterer, los creadores del término «etnografía», no por casualidad habían elegido palabras griegas para la formación del neologismo, ya que el historiógrafo griego Heródoto (ca. 485-ca. 424 a. C.) hasta en la actualidad es considerado como el fundador de la etnografía. Su obra estableció el modelo y varios tópicos de la etnografía de los humanistas del Renacimiento. Huellas de esta orientación en Heródoto se encuentran en la predilección de los etnógrafos de la primera época del descubrimiento por acentuar en sus descripciones las peculiaridades de las regiones y de sus habitantes. Obviamente, la temprana etnografía de las Américas se interesaba más en lo singular, extraordinario y maravilloso del Nuevo Mundo y, en menor grado, en los rasgos culturales comunes entre pueblos europeos y americanos. Siguiendo a Heródoto, por consiguiente, mucho menos atención

3 En el presente la noción «etnografía» tiene dos vertientes: por un lado también significa una descripción de una cultura, pero por otro lado se refiere a la recolección de datos mediante los diversos métodos etnográficos elaborados por la antropología (Gareis 2006: 592). 
se otorgó en las descripciones renacentistas a los paralelos etnográficos que a las extrañas costumbres de los pueblos americanos, de animales fantásticos y de seres monstruosos, como por ejemplo hombres con cabezas de perros, los cuales había ya descrito el famoso etnógrafo griego (Landfester 2000: 8-11, 15-18, 22 y ss.). Entre las maravillas del Nuevo Mundo, los primeros etnógrafos detectaron también otras representaciones de alteridad mencionadas en la obra de Heródoto, especialmente las belicosas amazonas y los «androphagoi», «los que comen hombres». Sin embargo, los etnógrafos renacentistas frecuentemente recurrieron a textos de autores posteriores, en especial a la Naturalis historia de Plinio el Viejo (23/24-79). En su obra los «androphagoi» de Heródoto que supuestamente comieron hombres (individuos masculinos) se convirtieron en «antropófagos», los cuales se alimentaron de carne humana. Otras temáticas inspiradas por autores de la Antigüedad fueron el discurso de los bárbaros y el de los esclavos ambos originados por Aristóteles (384-322 a. C.), como también los tópicos de la Edad de Oro y de la imposibilidad de habitar la así llamada «zona tórrida», es decir la zona intertropical o la franja ecuatorial (Gareis 2014: 358-359).

Si bien los autores de la primera época del descubrimiento esperaban encontrar en tierras americanas las maravillas que habían conocido en los textos antiguos, esto no significaba que el «otro», es decir el culturalmente otro, siempre fuera concebido como malo, monstruoso o inculto e inferior. La etnografía americana temprana más bien estableció una dicotomía entre salvajes buenos, inocentes y mansos por un lado y los «indios» feroces, malos y a veces incultos o bárbaros por otro lado. Esta dicotomía ya fue introducida por Cristóbal Colón (ca. 1451-1506) en sus primeros escritos sobre la población indígena de las Indias. En la relación de su primer viaje hizo una clara distinción entre los taínos, habitantes de las Antillas Mayores, mansos, pacíficos e inocentes y los caribes de las Antillas Menores, los que supuestamente eran belicosos, feroces y en la opinión de Colón por lo tanto superiores a los taínos (Gareis 1992: 
221). Colón afirmó incluso que los caribes debían ser gente de razón, ya que tenían armas y eran temidos como grandes guerreros por los taínos (Colón 1989: 62). Más tarde, se prolongaría la dicotomía entre indígenas buenos, cultos y los que eran considerados como malos, feroces o incultos en numerosas etnografías de pueblos americanos (Gareis 2017b: 134). Así, por ejemplo, el francés Jean de Léry (1536-1613) contrastaba en sus descripciones de la costa brasileña los cultos tupinambás de los bárbaros ouetacas (Léry 1578: Cap.V, 52-53, Cap.XV, 237 y sgs.; Gareis 2017b: 146). Semejante oposición también resalta entre los incas, creadores de un estado perfecto y los belicosos y salvajes chiriguanos en las obras del Inca Garcilaso y de otros autores que trataban del Perú (Garcilaso 1991: Lib.VII cap. XVII: 459-461; Guamán Poma 1980: 913 [f. 982]).

Además de temáticas y motivos adoptados de la Antigüedad griega y romana, los etnógrafos renacentistas también se valían del estilo y de los métodos de los autores antiguos, como el de la «autopsia», es decir, relatar lo que el autor había visto con sus propios ojos (Gareis 2014: 360). No es sorprendente por eso que varios autores de etnografías tempranas insistieran en referirse a hechos que ellos mismos habían presenciado y observado. Así, por ejemplo, afirmó André Thevet (1516-1590) en su libro Cosmographie de Levant [1554] que su contribución al conocimiento de esta región ya descrita por varios autores antiguos consta en sus propias observaciones. De manera que su texto presentaba nuevos datos gracias a la información que había reunido durante su viaje a esta región (Thevet 1556: 3 y sgs.). En el proemio proseguía con esta argumentación remitiéndose a Aristóteles (Thevet 1556: 13). Su contemporáneo Jean de Léry, famoso etnógrafo de los tupinamba de la costa brasileña, cuyo libro Claude Lévi-Strauss llamaría «el breviario del antropólogo», igualmente resaltó la importancia de la autopsia. ${ }^{4} \mathrm{~A}$

4 Claude Lévi-Strauss no solamente calificaba el libro de Jean de Léry «Histoire d'un voyage...» de ser «le bréviaire de l'ethnologue» (1955: 87), sino también lo 
diferencia de Thevet, a quien critica por su pretensión de ser testigo ocular si en realidad esto no era verdad, Léry asevera que él solamente quiere referir lo que él mismo había experimentado, visto, oído y observado personalmente (Léry 1580: Bvi, Cij-iij, Cap. I, 2). Es decir, que los etnógrafos renacentistas se asemejan bastante a sus colegas recientes al reclamar la autenticidad de sus datos a raíz de haberlos obtenido mediante su trabajo de campo.

A mediados del siglo XVI, por consiguiente, la etnografía ya no buscaba sobre todo lo maravilloso y lo singular en América como en la primera época después del descubrimiento, sino más bien enfocaba la descripción de lo que los autores habían visto en sus viajes, acercándose a la etnografía moderna. Aunque el título: Les singularitez... [1557], de la obra de Thevet sobre los habitantes de la costa brasileña todavía hace referencia a las singularidades de la tierra del Brasil, no se trata ya de describir en primer lugar monstruosidades y maravillas que supuestamente caracterizaban esta región, sino particularidades del clima, de la naturaleza o de las costumbres de sus habitantes (Thevet 1558). Siguiendo el modelo de la cosmografía antigua, Thevet intentaba dar una descripción completa de la «France Antartique», la colonia francesa en el Brasil, es decir que incluía capítulos sobre la flora y fauna, además de otros elementos característicos de esta tierra. Inspirándose en la Naturalis historia de Plinio el Viejo, Thevet compara las costumbres de los indígenas brasileños con las de la Antigüedad. De modo que al ilustrar constantemente las costumbres de los brasileños con las de la Antigüedad griega y romana, actualiza en cierta manera las informaciones etnográficas de los antiguos (Lestringant 1991: 107-110).

Las constantes referencias de Thevet a Plinio y al erudito renacentista Polidoro Virgilio (hacia 1470-1555) sirvieron en buena par-

consideró en general uno de los más importantes autores, remarcando además que las descripciones etnográficas de Léry habían ejercido mucha influencia sobre su propio trabajo antropológico (Lévi-Strauss 1982: 70). 
te a demostrar su conocimiento de los autores antiguos y a rechazar las críticas de algunos contemporáneos que pusieron en duda su formación humanista (Lestringant 1991: 13-15). Sin embargo, este intento se vio hasta cierto punto frustrado por el hecho que Thevet había delegado el trabajo de proveer las citas correspondientes al traductor y escribano M. Héret, el cual luego lo haría público (Lestringant 1991: 101-104, 107). De todos modos, la obra de Thevet puede servir como ejemplo de una típica descripción etnográfica de la mitad del siglo XVI (Gareis 2014: 363).

\section{VIDA Y FORMACIÓN}

José de Acosta nació en el año 1540 en Medina del Campo, el menor de una familia de nueve hijos. Su padre Antonio era un mercader acomodado y benefactor de la Compañía de Jesús. Cuatro de los cinco hijos varones ingresaron a la Compañía y dos de sus tres hermanas fueron religiosas (Burgaleta 1999: 3, 6-7). Los jesuitas fundaron su casa en Medina del Campo en 1551 y tan sólo un año después, en septiembre del 1552, José, no habiendo cumplido aún los doce años, se escapó de la casa para ingresar a la orden en Salamanca. Dada la poca edad de su hijo, los padres probablemente no hubieran asentido a esta decisión. El noviciado lo pasó de 1552 al 1554 en la casa de los jesuitas en su ciudad natal de Medina del Campo (Burgaleta 1999: 8-10). Durante su noviciado y en los años siguientes, José de Acosta recibió una educación humanista según el modelo establecido por los fundadores de la orden. El estudio comprendía en primer lugar tanto la gramática de los idiomas griego y latín como la literatura de los clásicos para formar a los estudiantes en el arte de la retórica de manera que pudiesen llegar a «eloquentia perfecta». Esta formación humanista tenía como principal objetivo aumentar la fuerza persuasiva de la retórica de los jesuitas, ya sea como religiosos en la península ibérica o como misioneros en el vasto imperio español (Burgaleta 1999: 12-13). 
A partir del año 1557, el joven Acosta estaría viajando a varios colegios jesuitas de Castilla y de Portugal para enseñar y completar sus estudios. A finales del 1557 y principios del 1558 estudió dos meses filosofía en el colegio de Coimbra. Es probable que en esta época naciera su interés por la misión al tener noticias de las experiencias de misioneros regresando de las colonias portuguesas, puesto que los jesuitas poco después de la fundación de la orden ya mandaban a los primeros misioneros a las posesiones portuguesas de ultramar (Burgaleta 1999: 14-15). La Corona española, en cambio, admitió recién en 1566 que misioneros de la Compañía de Jesús emprendiesen su labor en América, mientras que las otras órdenes misioneras de los dominicos, franciscanos, mercedarios y agustinos habían obtenido los respectivos permisos anteriormente (Konetzke 1974: 248-261). Según una carta de Acosta dirigida al Padre General en 1569, estaba anhelando ir a las misiones de América desde hacía nueve años, periodo que coincide con su estadía en Portugal (Burgaleta 1999: 15).

En otoño del 1559, José de Acosta emprendió estudios de filosofía y teología en la universidad de Alcalá de Henares, siguiendo los cuatro años de los cursos primero de filosofía entre 1559 y 1563 y luego los de teología entre 1563 y 1567. Este mismo año de 1567 tuvo lugar su ordenación y en 1570 hizo los votos finales. Al año siguiente finalmente se cumplió su deseo de integrar las filas de los misioneros al obtener permiso de ir al Perú. Zarpó su barco para las Indias en junio de 1571 y, después de una escala de varios meses en Santo Domingo, llegó a Lima en abril de 1572 (Burgaleta 1999: 1531). Ya al año siguiente, el provincial de los jesuitas le mandó hacer una visita por el sur del virreinato. Durante un año y medio Acosta tuvo ocasión de conocer a fondo todas las ciudades principales de la colonia. Su viaje le llevó al Cusco, Arequipa, Potosí, La Paz, Chuquisaca entre otros lugares, en donde podía recolectar datos etnográficos y reunir información sobre diversos fenómenos naturales y culturales característicos de los Andes, además de adquirir conocimientos del idioma quechua (Burgaleta 1999: 36-37; Gareis 
2001: 2-3; Marzal 1993: 93). En 1576, siendo ya superior provincial, emprendió un segundo viaje al sur, en el cual fundó la casa de Juli a orillas del lago Titicaca (Marzal 1993: 93). Un tercer largo viaje le llevó en el año 1578 por el interior del Perú, en donde tomó más notas, las que luego formarían parte de su gran obra Historia natural y moral (Del Pino 2009: 110; Gareis 2017a: 26). Dejó el cargo de provincial en 1581, pero pronto le tocó otro rol importante como teólogo consultor en el Tercer Concilio Limense entre 1582-1583. Muchos decretos del concilio ostentan paralelos con las ideas acerca de la misión y buena doctrina expresadas por Acosta en su obra De Procuranda. Al igual, es notoria su influencia en la redacción del catecismo trilingüe y en el Confessionario para los curas de indios, ambos publicados en 1585 en Lima (Del Pino 1992: 310-312; Marzal 1993: 93). Permaneció en total catorce años en el Perú hasta 1586, cuando regresó a España pasando por México, en donde se quedó casi un año. Allí aprovechó para recoger información adicional sobre los aztecas y otras culturas mexicanas para su libro Historia naturaly moral (Burgaleta 1999: 39-53; Marzal 1993: 93-94). En 1587, de regreso en España, desempeñó varios puestos en la Compañía y un papel importante en el futuro de la orden en su tierra natal, pero también en el Perú. Publicó su obra De Procuranda Indorum Salute en 1588 y dos años después salió de la prensa su libro magistral Historia natural y moral. A principios del 1597 asumió su último cargo como rector del colegio jesuita de Salamanca. Murió en febrero del 1600 en la misma ciudad (Burgaleta 1999: 56-69).

\section{EL JESUITA NATURALISTA, ETNÓGRAFO, ANTROPÓLOGO E HISTORIADOR}

La orientación humanista del currículo de los colegios jesuitas se plasma en las obras de José de Acosta, tanto en la parte naturalista y etnográfica como también en el acercamiento teórico (Del Pino 2016: 38, 41). Sin embargo, a diferencia de las etnografías re- 
dactadas a mitad del siglo XVI, como por ejemplo las de Thevet y Léry, Acosta ya no utiliza los escritos de los autores de la Antigüedad meramente para demostrar su erudición o como una cantera de sacar ejemplos para la comparación con las culturas americanas. Tampoco le interesaba solamente refutar las teorías antiguas (Gareis 2014: 363). Quería, más bien, averiguar a fondo la causa de los fenómenos y llenar vacíos respecto a diferentes problemas que todavía no habían sido tratados en la bibliografía (Acosta 1590: Proemio, 9-10). Acosta, por lo tanto, no se contentó con la simple compilación de información sobre la naturaleza, datos etnográficos o con un recuento de relatos, sino intentaba estudiar todos estos fenómenos más a fondo de lo que hasta en aquel entonces se había hecho de manera que su obra podría contribuir una nueva visión de las tierras y culturas americanas. El último fin de su Historia naturaly moral lo define en el deseo de mostrar a través de su libro la grandeza y maravilla de la creación de Dios (Acosta 1590: Proemio, 10-12).

Vale decir que aunque por el título «Historia natural y moral» su obra podría parecer siguiendo al modelo de la Historia naturalis de Plinio, el texto de Acosta en ninguna parte es una mera compilación de datos como lo son los 37 libros del autor romano. Muy al contrario de los objetivos de Acosta, Plinio quería ofrecer con su obra enciclopédica la totalidad de las informaciones y todo el saber que hasta entonces se había conocido (Plinius 1973: lib. 1, párrafos 1218). Es posible ver un paralelo, sin embargo, en cuanto a la declaración de Plinio que el contenido de su obra es «la naturaleza, es decir el mundo real». ${ }^{5}$ Dada la principal intención de Acosta de exaltar la creación divina a través de la descripción del mundo, era desde luego necesario presentar ante todo la información disponible en este momento para poder luego analizar y discutir los fenómenos detalladamente. De acuerdo con este propósito, los primeros cuatro libros de la Historia natural y moral están dedicados a los fenómenos

5 «[... rerum natura, hoc est vita, narratur [...].» (Plinius 1973: Lib. 1, párrafo 12). 
naturales del Nuevo Mundo, mientras que los tres restantes libros se ocupan de las «costumbres y hechos» de los pueblos americanos (Acosta 1590: Prólogo, 300). Los dos primeros libros que tratan de fenómenos naturales, ya los había escrito en latín en 1577, estando todavía en el Perú (MacCormack 1991: 255). ${ }^{6}$ Con el título De Natura Novi Orbis, los antepuso como prólogo a su tratado De Procuranda Indorum Salute publicado en 1588. Ya de vuelta en España, los tradujo al español y completó la parte de la historia natural con otros dos libros (Gareis 2001: 4).

El primero de los cuatro libros sobre la historia natural discute una multitud de teorías de autores cristianos y antiguos sobre diversos problemas tocantes a la naturaleza del cielo (Acosta 1590: Lib. I, caps. 1-5, 13-27). Ya en estos primeros capítulos de su historia se nota el método científico de Acosta, muy distinto de la forma como los autores renacentistas de las generaciones anteriores se habían acercado a estos fenómenos. Acosta no trabaja con la comparación, ni con la analogía como lo hicieron sus antecesores en los escritos sobre las Américas. Más bien, el jesuita erudito y naturalista examina, observa y constata, de modo que aporta elementos de análisis y de conocimiento invaluables para el futuro. En cierta manera proponía con su acercamiento un redescubrimiento pragmático de América, basado en una visión científica bastante moderna (Courcelles 2003: 312-313). Se hace notar tanto en los primeros libros que tratan de la complexión física del Nuevo Mundo, como también a lo largo de su obra que Acosta no duda en criticar y descartar teorías de autores antiguos, ni tampoco afirmaciones de la biblia o de escritores religiosos eminentes, tal como san Agustín. Es decir que José de Acosta a través de la discusión de las teorías existentes sobre los fenómenos en cuestión, propone una mirada fresca sobre

6 Según Fermín del Pino, Acosta envió el informe geográfico De Natura Novi Orbis en 1582 al general Claudio Aquaviva como complemento del tratado De Procuranda Indorum Salute, el que ya había enviado anteriormente en el año 1576 (Del Pino 2016: 41). 
la problemática, además de tomar una posición independiente en la interpretación de los datos (Gareis 2001: 1-3).

La Historia de Acosta sigue, sobre todo en la primera parte tocante a la historia natural, el plan de una cosmografía de la época y también se habrá inspirado especialmente en cuanto a la descripción de la naturaleza americana en las Relaciones geográficas efectuadas por oficiales en ultramar por orden de la Corona. En 1577 se había concebido un catálogo de 50 preguntas que abarcaban desde la historia de la región, las tradiciones culturales de sus habitantes, la flora y fauna, hasta los yacimientos de metales y otras muchas temáticas. Juan López de Velasco, en aquel entonces cosmógrafo y cronista oficial del reino, jugaba un papel importante en la redacción del cuestionario (Gareis 2003: 228-229). De manera parecida, Acosta apunta a dar una descripción completa de los fenómenos naturales del Nuevo Mundo. En adición a la temática de la posición de los cuerpos celestes, con la cual prosigue el primer libro de la Historia, también discute la afirmación de varios autores del pasado sobre que no puedan existir antípodas y de cómo era posible que se engañaron en estas cuestiones (Acosta 1590: lib. I, caps. 3-10, 19-43). Luego se dedica a responder a la pregunta de qué forma los primeros habitantes habían llegado al continente americano, adelantando su teoría de la población de América por tierra (Acosta 1590: lib. I, cap. 20, 68-84). El segundo libro abre con la discusión de otro tópico de la historia natural, el de la zona tórrida inhabitable según los autores de la Antigüedad. Opinión que Acosta (1590: lib. II) rebate mostrando los errores inherentes a esta suposición. ${ }^{7}$ Los libros tres y cuatro continúan con la descripción primero de los vientos, del mar, de lagos, de ríos, de la calidad de la tierra tomando en cuenta

7 Del tópico de la así llamada «zona tórrida», es decir de la zona intertropical supuestamente inhabitable según los escritores antiguos, también se ocuparon otros autores de la época, como el ya mencionado futuro cosmógrafo del rey de Francia, André Thevet, en su descripción de la colonia francesa en el Brasil (Thevet 1558: cap. 18-19, fs. $34^{\mathrm{r}}-37^{\mathrm{v}}$ ). 
algunas peculiaridades del Perú. ${ }^{8}$ Concluye el tercer libro con una reflexión sobre los volcanes, temblores de tierra y terremotos. Se presentan por ende al modo de la filosofía griega los cuatro elementos simples (no compuestos): aire, agua, tierra y fuego (Del Pino 2016: 49). Prosigue el libro cuarto con los elementos compuestos, en especial los metales que se encuentran en América, de qué manera los indígenas los labran y de diferentes técnicas de extraer la plata del mineral mediante hornillos o con el método de amalgamación con mercurio introducido por los españoles. Entre los tesoros que se pueden encontrar en América figuran también las piedras preciosas y perlas. Los restantes capítulos del libro cuatro describen la flora y fauna, tanto de plantas y animales salvajes como también de las especies domésticas, incluyendo en el último capítulo una relación de la naturaleza y virtudes de las piedras bezoares utilizadas en la medicina de su época. Al resumir las temáticas de la primera parte de la Historia, se perfila el orden aristotélico de la descripción, pero sin que Acosta hubiera seguido o se hubiera aferrado ni al autor griego, ni tampoco a la patrística, sino que resalta también en este apartado sobre la historia natural su pensamiento independiente (O'Gorman 1989: 132-133, 137-140, 147-149).

La variedad de temáticas y la cantidad de información que ofrece esta primera parte de la Historia de José de Acosta demuestra su erudición y curiosidad, como también el afán de transmitir el conocimiento de estas «cosas nuevas» y las conclusiones a las que había llegado a los lectores en Europa con el fin de despertar su interés en los pueblos americanos y fomentar la comprensión de las peculiaridades de sus culturas. ${ }^{?}$

8 Fermín del Pino llama la atención sobre las consideraciones de Acosta relativas al viento del Sur y otras peculiaridades climáticas del Perú, hallazgos del jesuita que más tarde asombraron al científico alemán Alexander von Humboldt (Del Pino 2016: 49-50, 53, 55).

9 Acerca de la noción de «curiosidad» en esta época, véanse también las consideraciones de Fermín del Pino (2016: 42). 
Acosta también destaca por su aporte a la etnografía de los pueblos americanos, sobre todo del Perú y de México. A lo largo de sus dos libros, De Procuranda y la Historia, proporciona cantidad de datos etnográficos referentes a diversos fenómenos culturales. En primer lugar le interesaban las religiones precolombinas de los pueblos andinos y mexicanos, pero también se ocupaba de los calendarios, de sus costumbres, de su vida social, de su economía y organización sociopolítica. Además, trataba varias tecnologías y conocimientos indígenas, aportaba datos lingüísticos, y al reunir este cuerpo de datos etnográficos considerable, hace resaltar, al mismo tiempo, los logros de las culturas precolombinas de la América española (Gareis 2001: 4; Marzal 1993: 99-100).

Las informaciones etnográficas ofrecidas eran por una parte el fruto de sus propias observaciones, indagaciones y conversaciones con miembros de los diferentes grupos étnicos de la población colonial durante su estadía en el Perú. Por otra parte, tenía acceso a datos etnográficos en los informes enviados desde las diversas casas con sus colegios y de las doctrinas de los jesuitas en la vasta provincia del Perú. Durante su provincialato entre 1576 y 1581 le correspondía además de redactar las así llamadas «cartas annuas», es decir, los informes anuales que todas las provincias jesuíticas habían de enviar al general de su orden. ${ }^{10}$ Mediante estos documentos, Acosta se enteraba de particularidades regionales, acontecimientos y datos etnográficos. Una idea de la variada información que se podía sacar de estos textos la dan las citas insertadas en sus cartas anuales. Dada la naturaleza de estas fuentes, la mayoría de los datos se referían a temas religiosos. En adición a todos estos datos, compiló más información etnográfica de una multitud de fuentes escritas (Del Pino 2009: 210-211; Gareis 2001: 2-3). Entre otros textos a los que Acosta tenía alcance durante su estadía en el Perú, figuraron los escritos de

10 Acerca de las Cartas annuas y su valor como fuentes etnográficas, véase Gareis (1990: 613-615; 2017a: 26-27, 30-31). 
Juan de Matienzo y de Polo de Ondegardo, dos oficiales de la administración colonial. En su papel de consultor del Concilio Limense, Acosta elaboró una versión del tratado de Polo de Ondegardo sobre las religiones andinas para la publicación en el Confessionario para los curas de indios, que salió de la prensa en 1585. Este tratado que anteriormente ya había circulado en el Perú en forma manuscrita, adquirió con la publicación suma importancia como fuente utilizada por varios autores coloniales (Gareis 2003: 197-200).

Si bien los datos etnográficos que aporta José de Acosta sobre los pueblos americanos forman un cuerpo de saber importante, no fue solamente por eso que se le otorgó tanta atención en el pasado y también en la actualidad. La etnografía, aunque en efecto concede a sus escritos un valor por sí mismos porque satisface la curiosidad de los lectores, era ante todo la base sobre la cual Acosta podía discutir y concebir sus ideas teóricas acerca de los hombres americanos y los caminos para su salvación.

Acosta presenta diversas teorías antropológicas en sus dos obras mayores, de las cuales la primera responde a la cuestión de qué manera llegaron los primeros hombres al continente americano. $\mathrm{Al}$ igual que a lo largo de sus trabajos discute otras preguntas relativas al Nuevo Mundo y los pueblos americanos, también aquí expone la problemática con peculiar claridad. De modo que ofrece a los lectores todas las informaciones acerca del problema discutido, presentando luego sus hipótesis, dándoles así la posibilidad de formarse ellos mismos una idea del asunto. Con respecto a la población del continente americano, señala tres posibilidades, aunque pronto descarta que los primeros hombres hayan podido llegar navegando por mar dirigiéndose a tierras americanas intencionalmente, porque en esta época remota todavía no tenían los conocimientos necesarios para emprender un viaje tan largo por mar abierto (Acosta 1590: lib. I, cap. 16, 56-61). Dedica los dos siguientes capítulos del primer libro de su Historia a demostrar que en aquel entonces no se disponía de los instrumentos de navegación como en su época, por lo cual 
si han llegado por mar, podría haber sido solamente involuntariamente a causa de una tormenta que los habría echado a tierra en un naufragio (Acosta 1590: lib. I, caps.17-19, 61-68). Así que nuestro autor llega a la conclusión que los primeros hombres no hubieran podido llegar a América sino por tierra o, si acaso vinieron por mar, entonces debe haber sido atravesando un estrecho en el norte del continente (Acosta 1590: lib. I, caps. 20-21, 68-75). Refuta tanto la hipótesis del origen de los indígenas americanos de la isla Atlántida, como también la del origen judío. La primera, por parecerle demasiado fantástica, y la segunda porque los judíos usaban letras y la mayoría de los pueblos americanos no. Acosta también consideraba inverosímil que siendo descendientes de judíos se hubiesen olvidado de su religión y costumbres por completo (Acosta 1590: lib. I, caps. 22-23, 75-80). Por todo esto afirma:

Mas assi a bulto y por discrecion podemos colegir de todo el discurso arriba hecho, que el linage de los hombres se vino passando poco a poco, hasta llegar al nueuo orbe, ayuda[n]do a esto la continuidad, o vezindad de las tierras, y a tiempos alguna nauegacion [...]. (Acosta 1590: lib. I, cap. 24, 81).

Es decir que nuestro autor en ningún momento abandona la argumentación científica y nunca presenta conjeturas como hechos. Estos son, sin embargo, precisamente los puntos criticados por Helga Gemegah en su tesis doctoral, dedicada exclusivamente a discutir la teoría de José de Acosta de la población de América por tierra. Según la autora, Acosta siguió con su teoría del origen de los indígenas americanos de Asia la visión de la Corona española que con esta afirmación quería reivindicar su derecho a la posesión de las regiones asiáticas hasta entonces portuguesas o bajo influencia del Portugal (Gemegah 1999: 209-218). Aunque, a mi modo de ver, todo el argumento de esta autora no es ni convincente, ni tampoco respaldado por las fuentes históricas, es de notar que varios siglos después de su publicación todavía se le otorga tanta importancia a las obras de Acosta. Desde luego, mucho menos es lícito reprochar 
a José de Acosta que, al suponer que los primeros hombres vinieron de Asia a América, siga su razonamiento lógico basado en la convicción de la unidad del género humano, en vez de favorecer un origen poligenético de los hombres, como sugieren muchos mitos indígenas americanos. ${ }^{11} \mathrm{Si}$ bien la suposición del origen monogenético se funda en la doctrina católica, a lo largo de sus obras Acosta demuestra que no adopta todas las afirmaciones de la Biblia o de los santos padres de la Iglesia sin examinarlas previamente. Por eso, resalta claramente que no concibió su teoría para comprobar ni el derecho de la Corona española a las posesiones portuguesas en Asia, ni tampoco los hechos relatados en la Biblia o cualquier otra afirmación, sino, al contrario, su razonamiento más bien le sirve para encontrar respuestas al problema del origen de los hombres americanos. Además, su teoría monogenética significa que los pueblos americanos forman parte del género humano y por consiguiente no difieren en su condición humana de los hombres en otras regiones del mundo.

Otro tema que Acosta abarca en sus dos obras magistrales está en cierta forma emparentado con la teoría del origen de los pueblos americanos, puesto que según su opinión vinieron hace mucho tiempo pequeños grupos de cazadores, los cuales sólo después y poco a poco desarrollaron la diversidad cultural que los europeos encontraron en América (Acosta 1590: lib. I, cap. 20: 72; cap. 24, 81). Por primera vez se ocupa de la temática en el proemio de su primera gran obra De Procuranda, al reflexionar sobre la dificultad de

11 Gemegah está convencida que la teoría del origen de los pueblos americanos elaborada por Acosta era una de las más graves «falsificaciones» de la historia, ya que ellos mismos afirmaban haber siempre estado allí. Según Gemegah, los arqueólogos están equivocados en seguir la teoría de Acosta, en cambio deberían más bien dedicarse a buscar pruebas de un origen americano de los pueblos nativos (1999: 215-218). Obviamente, esta autora no acepta que la teoría de Acosta - desde luego adaptada a los conocimientos científicos modernos- fue comprobada por hallazgos arqueológicos y es todavía vigente en la actualidad (Marzal 1993: 103). 
adaptar los métodos de evangelización a los diversos pueblos americanos, pues afirma que «[...] son muy varias las naciones en que están divididos, y muy diferentes entre sí [...]» que lo hace prácticamente imposible de encontrar un método que sea aplicable a todas las culturas (Acosta 1952: 43). Desarrolla en lo siguiente una tipología de evolución cultural al distinguir tres grupos de «bárbaros»: El primer grupo incluye los chinos y semejantes culturas, mientras la segunda y tercera categoría de bárbaros estaba integrado de pueblos americanos. A los pueblos de la segunda categoría les faltaba el «uso de escritura», sin embargo, contaban con una organización socio-política bien ordenada, con asentamientos estables, leyes y un culto religioso. A este grupo pertenecían los antiguos peruanos y mexicanos. En cambio, el tercer tipo de «bárbaros» vivía «sin ley, ni rey»y por ende eran considerados «salvajes» (Acosta 1952: 46-48). Esta teoría era probablemente la que ejerció más influencia en autores posteriores y estaba vinculada a la cuestión de la justa guerra. Acosta dedica varios capítulos del libro segundo de De Procuranda a esta pregunta. Después de haber refutado la mayoría de las razones alegadas por los españoles por hacer la guerra a los pueblos americanos, explica en el capítulo quince «Cuándo sea lícito hacer la guerra a los infieles». En suma llega a la conclusión que la guerra a los pueblos «bárbaros» tan sólo es justa «cuando el príncipe empuña las armas provocado por injurias» (Acosta 1952: lib. II, cap.15, 199).

Acosta discute el tema de la diferente evolución de los pueblos americanos también en la Historia. Sin embargo, aquí lo hace a través de su organización política. Distingue entre las sociedades indígenas «tres generos de gouierno, y vida enlos Indios». Según el esquema de Acosta, el primer género lo integran las monarquías, el segundo, las behetrías, es decir los pueblos organizados en cacicazgos, y el «terçer genero de gouierno es totalmente barbaro, y son Indios sin ley, ni Rey, ni assiento» (1590: lib.VI, cap.19, 430). Como ejemplos del primer género menciona los incas del Perú y los aztecas de México. Los pueblos de la segunda categoría de «be- 
hetrías» se gobernaban por consejos. En tiempo de guerra elegían un cacique y todo el pueblo obedecía sus órdenes. En esta categoría de grupos organizados en cacicazgos, Acosta hace referencia a los mapuches de Chile y para los pueblos incultos y salvajes del tercer género nombra, entre otros muchos, los chiriguanos de las tierras bajas al oriente de los Andes. Aunque Acosta hace igualmente una distinción entre diversos tipos de culturas indígenas, ya no presenta la visión bipolar que encontramos en las relaciones de la primera época del descubrimiento, la cual distinguió entre «indios buenos y mansos vs. indios malos y salvajes belicosos». Su esquema supera esta bipolaridad inicial de la imagen de los indígenas americanos y al mismo tiempo abre otra temática relacionada con las diferentes organizaciones de estos pueblos. Entre otros problemas se ocupa de la pregunta de por qué la colonización y evangelización fue más fácil con los pueblos americanos del primer género que con otros del segundo o tercer género. Según Acosta (1590: lib. VII, cap. 28: $530,532)$, en los estados de los aztecas y especialmente en el de los incas, la organización política centralizada, la introducción del quechua como lengua estatal y otros rasgos característicos de estas monarquías ya habían preparado el camino para los españoles. En cambio, los pueblos de la segunda categoría, organizados en cacicazgos, como los mapuches de Chile y los de la tercera categoría, como los chichimecas de México o los chiriguanos y otros semejantes grupos étnicos, se opusieron a los españoles en una resistencia enconada. Acosta llama la atención sobre la dificultad que los españoles encontraron en los pueblos que «no reconocen vn Señor» (Acosta 1950: lib. VII, cap. 28, 530). A su modo de ver, el mayor problema de tratar con aquellas sociedades, reside en el hecho que no tenían autoridad central con la que misioneros o militares podrían acordar un convenio. Así, las tropas españolas nunca tuvieron éxito en someter a los mapuches de Chile, al igual que fallaron en conquistar los chichimecos de México y otros pueblos de este tipo (Acosta 1950: lib. VII, cap. 28, 531-532). 
La tercera temática a la que Acosta se dedica en sus obras enfoca las dificultades de llevar a cabo la misión entre los indígenas americanos. En la raíz del mal está, en su opinión, el diablo que trata de impedir la salvación de estos pueblos. Acosta se ve confirmado en su interpretación por los paralelos etnográficos que encuentra con mayor claridad en algunos ritos religiosos de los pueblos andinos que se asemejan mucho a los respectivos sacramentos cristianos, sobre todo la comunión y la confesión. Según Acosta, el diablo quería imitar a Dios y ser venerado de igual manera, por lo cual introdujo estos sacramentos. ${ }^{12}$ Por un lado, la perversión de los ritos católicos por el diablo tenía también algo bueno, puesto que facilitó la labor de los misioneros porque los indígenas ya conocían los conceptos básicos de estos sacramentos (Acosta 1952: lib. VI, cap.12, 563-564; 1590: lib. V, caps.23-27, 358-381). Por otro lado, pone en claro que siendo el diablo la principal causa de la idolatría (Acosta 1590: lib. V, cap.1, 303-305), los pueblos americanos no son ni «faltos de entendimiento», ni tampoco incapaces de recibir la palabra de Dios o de convertirse a la fe católica (Lib. VI cap.1: 395-397). Es decir, Acosta no pone en duda en ningún momento la capacidad de los pueblos americanos de recibir la fe, una vez que estén liberados de las garras del diablo. Otra razón por la que la evangelización de los indígenas americanos todavía no rindió fruto como se había esperado se ha de buscar en las faltas de doctrineros y en la violencia, explotación y agresión ejercidas por los españoles en detrimento de la población indígena. A lo largo de su libro De Procuranda no se cansa de señalar cuánto el mal comportamiento de los españoles daña a los indígenas y, por consiguiente, impide su salvación. Así, por ejemplo, dedica un capítulo entero a mostrar que «hay grande esperanza de

12 En cuanto a las maquinaciones del diablo y el rol que se le atribuía en la interpretación de las religiones americanas por los contemporáneos, véanse Marzal (1993: 98) y sobre todo MacCormack (1991: 249-281), quien dedica todo un capítulo de su libro al análisis y a la discusión de esta problemática con relación a la obra de Acosta. 
verdadera fe y salvación para los indios, y es contrario al espíritu de Dios sentir lo contrario» (Acosta 1952: lib. I, cap. 15, 115). Además, afirma que está convencido que la salvación de los indígenas hasta el momento en mayor parte había sido impedido por la incapacidad y la codicia de los doctrineros, los que sólo buscan su interés personal, en vez de dar buen ejemplo a los feligreses indígenas (116). En adición a los malos doctrineros también inculpa a los españoles de maltratar y robar a los indígenas para apropiarse de sus haciendas (cap.13, 109-110; lib. II, cap.18, 209-210).

Es de notar que la versión publicada de su obra De Procuranda había sido sometida a la censura. Vale decir que nosotros tan sólo conocemos la versión expurgada por orden de Claudio Aquaviva, a la sazón general de los jesuitas. En 1582 escribió al provincial Gil González que el manuscrito «Pro indorum salute curanda» de José de Acosta le había gustado bastante, pero que era imprescindible antes de la impresión quitar ciertos pasajes del texto que daban cuenta de las crueldades de los conquistadores (citado en Egaña 1954-1970, t. III, doc. 46: 195-196). Todo esto indica que la crítica de Acosta al comportamiento de los españoles había sido mucho más enérgica en la versión original de su obra. En la Historia declara de antemano que no piensa escribir sobre los hechos de los españoles en la empresa de América porque ya todo esto es sabido. Más bien, le interesa enfocar las cosas del Nuevo Mundo y las sociedades indígenas (Acosta 1590: 301).

Para José de Acosta tanto los fenómenos de la naturaleza, es decir, la historia natural, como también la historia moral, comprendiendo los hechos y costumbres de los hombres, formaban parte de la historia universal. Esta, a su vez, era para Acosta equivalente a la historia de la salvación. Al dar a conocer la historia natural y moral del continente americano, Acosta inscribió el Nuevo Mundo y sus habitantes en la historia universal del género humano, asignándoles así un lugar en el seno de la humanidad. 


\section{Epílogo: Recepción e influencia de la obRa de José de Acosta}

Las dos obras magistrales de Acosta tuvieron muy buena acogida entre los contemporáneos. De Procuranda, aunque según Marzal (1993: 94-95) por varios motivos no puede considerarse un texto definitivo, ejerció mucha influencia como manual de teología pastoral. Salió por primera vez en 1588 y al año siguiente hubo una segunda edición en Salamanca. En 1596 se publicaron una tercera edición en Colonia y una cuarta en Lyon. La quinta edición salió recién en 1858 en Manila y en el siglo XX hubo otras dos ediciones en España. Francisco Mateos publicó una traducción al español en 1952 en Madrid y en el mismo lugar salió dos años después la séptima edición de la obra en la colección Biblioteca de Autores Españoles (Marzal 1993: 94). Uno de los autores que se inspiraron en De Procuranda fue Alonso de Peña Montenegro, obispo de Quito, quien publicó en 1668 un manual para párrocos de comunidades indígenas (Gareis 2003: 204).

Aún más éxito tuvo la Historia de Acosta. Al año siguiente de la primera edición en Sevilla en 1590, se publicó en Barcelona por segunda vez. Luego siguieron ediciones en Madrid (1608, 1792, 1894) y ya en el siglo XX dos en la ciudad de México $(1940,1962)$, entre varias otras (Marzal 1993: 101). La más reciente publicación de la Historia se debe a Fermín del Pino, quien elaboró una nueva edición crítica en 2008 (Del Pino 2009: 110). Además, se publicaron traducciones a muchas lenguas europeas: la primera en 1596 al italiano, en 1597 al francés, la que tuvo reimpresiones en 1600, 1606 y en 1616. Otras traducciones fueron al holandés en 1598 y en 1624 (Valdeón 2014: 193), como también al alemán en 1598 (Gareis 2001: 5), seguidas por otras en 1601 y 1617 (Del Pino 2009: 111; Valdeón 2014: 193). Versiones en latín se publicaron en 1602 y 1603 y la primera traducción al inglés salió de la prensa en 1604 (Valdeón 2014: 193).

Basta ver la gran difusión de la Historia para formarse una idea de la enorme influencia que la obra tuvo sobre autores contempo- 
ráneos como también sobre los de siguientes generaciones. Fermín del Pino atestigua que este libro «se trata de una de las obras más leídas en su tiempo sobre el Nuevo Mundo y sus habitantes» (1992: 313; 2009: 111). Además, afirma que actualmente es la crónica de Indias más reeditada, lo que atribuye al hecho de que Acosta, a diferencia de los otros autores de su época, no se interesó por la historia de los europeos en América, sino se dedicó exclusivamente a tratar de la naturaleza y hombres americanos (Del Pino 2009: 111). En adición a esto, adquirió gran importancia por la variedad de temáticas abordadas y la diversidad de problemas tratados. Esto, como también la erudición de Acosta y su seriedad científica, le valieron la atención de lectores de diferentes disciplinas, países y épocas. Entre los autores contemporáneos que citaron al padre Acosta destaca el Inca Garcilaso, cuya obra tuvo igualmente gran difusión por ser traducida a varios idiomas europeos y por su cantidad de reimpresiones (Brading 1991: 290, 416; Gareis 2003: 198-199). Es decir que la Historia de Acosta alcanzó todavía más lectores a través de los Comentarios del Inca Garcilaso.

En el siglo XVII, la obra de Acosta despertó el interés de dos teóricos ingleses del estado. Thomas Hobbes (1588-1679) en su obra Leviathan (1651) algunas veces se refiere a los «pueblos salvajes de América» o también a los «idólatras americanos», lo que hace pensar en las sociedades sin autoridad central de acuerdo a las ideas de Acosta sobre la evolución de los sistemas políticos en el Nuevo Mundo (Brading 1991: 471; Del Pino 1992: 313-314; Gareis 2001: 5). Sin embargo, Hobbes no da las citas correspondientes. John Locke (1632-1704), en cambio, cita los datos de Acosta en sus «Dos tratados sobre el gobierno civil» (1689). Al igual que Hobbes, Locke (1988: párrafos 101-102, 105) también se interesa sobre todo por los «hombres en su estado natural», es decir por las sociedades sin organización estatal. Aunque estos autores últimamente llegan a diferentes conclusiones con respecto a la mejor forma de organización política, ambos teóricos basan su argumentación en 
«los salvajes americanos», es decir, en la tercera categoría de etnias americanas según Acosta. En el siglo XVIII, otros muchos autores recurren a las teorías de Acosta, como por ejemplo el jesuita francés Joseph-François Lafitau (1681-1746) para su obra sobre los iroqueses (Del Pino 1992: 314) o el presbiteriano escocés William Robertson (1721-1791), quien defendió la teoría del poblamiento de América concebida por Acosta (Brading 1991: 467-470). Finalmente, el erudito naturalista alemán Alexander von Humboldt (1769-1859) se refiere frecuentemente a la Historia de Acosta, sobre todo con relación a varios fenómenos de la naturaleza americana tratados por el jesuita (Del Pino 2016: 54-55). Aprecia la contribución de Acosta a la historia natural afirmando que era uno de los fundadores de la física del globo (Del Pino 2016: 55; Courcelles 2003: 317).

No es posible mencionar a todos los autores los que de una $\mathrm{u}$ otra manera habían sido influidos por los escritos de José de Acosta, pero es obvio que durante siglos e incluso hasta en la actualidad su obra presenta una fuente de inspiración para el mundo científico.

\section{REFERENCIAS}

Acosta, S. J., José de

1952 [1588] De Procuranda Indorum Salute. (Predicación del Evangelio en las Indias). Introducción, traducción y notas por Francisco Mateos (Colección España Misionera). Madrid: Consejo Superior de Misiones.

$1590 \quad$ Historia natural y moral delas Indias, en que se tratan las cosas notables del cielo, y elementos, metales, plantas, y animales dellas: y los ritos, y ceremonias, leyes, y gouierno, y guerras de los Indios. Compuesta por el Padre... Sevilla: casa de Iuan de León.

Brading, David A.

1991 Orbe indiano. De la monarquía católica a la república criolla, 1492-1867. México: Fondo de Cultura Económica. 
Burgaleta, Claudio M.

1999 José de Acosta, S.J. (1540-1600): his life and thought. Chicago: Loyola Press.

Colón, Cristóbal

1989 Textos y documentos completos. Relaciones de viajes, cartas y memoriales. Madrid: Alianza.

Courcelles, Dominique de

2003 «La conquête d'un savoir raisonnable: 1'Histoire naturelle et moralle des Indes, tant Orientalles qu'Occidentalles du P. jésuite José de Acosta, 1598». En: John D. Lyons y Cara Welch (eds.). Le savoir au XVII siècle. Actes du $34^{\circ}$ congrès annuel de la North American Society for Seventeenth-Century French Literature, University of Virginia, Charlottesville, 14-16 mars 2002. Tübingen: Gunter Narr Verlag, pp. 311-321.

Del Pino Díaz, Fermín

1992 «La Renaissance et le Nouveau Monde: José de Acosta, jésuite anthropologue (1540-1600)». L'Homme, vol. XXXII, n. 122-124, pp. 309-326.

2009 «Crónicas de Indias sin dibujos y con dibujos: el texto indiano de Acosta (1590) interpelado por los dibujos de De Bry (1601-1602).» En Fermín del Pino-Díaz, Pascal Riviale y Juan J. R. VillaríasRobles (eds.). Entre textos e imágenes. Representaciones antropológicas de la América indígena. Madrid: Consejo Superior de Investigaciones Científicas, pp. 105-132.

2016 «La tradición naturalista de algunos jesuitas en los Andes». Nuevas de Indias. Anuario del CEAC, I, pp. 34-60. DOI: http://dx.doi. org $/ 10.5565 / \mathrm{rev} /$ nueind.5

Egaña, Antonius de

1954-70 Monumenta Peruana. 5 vols. Roma: Apud «Monumenta Historica Soc. Iesu».

Garcilaso de la Vega, Inca

1991 [1609] Comentarios reales de los incas. Ed. por Carlos Araníbar. 2 vols., Lima: Fondo de Cultura Económica. 
GAREIS, Iris

1990 «La <idolatría andina y sus fuentes históricas. Reflexiones en torno a 〈Cultura andina y represión〉 de Pierre Duviols». Revista de Indias, vol. 50, pp. 607-626.

1992 «Kolumbus und Amerika: Mythenbildung und historische Wirklichkeit». En: Wolfgang Stein (ed.). Kolumbus oder wer entdeckte Amerika? München: Hirmer Verlag, pp. 211-226.

2001 «José de Acosta: Historia Natural y Moral de las Indias, en que se tratan las cosas notables del cielo, y elementos, metales, plantas, y animales dellas: y los ritos, y ceremonias, leyes, y govierno, y guerras de los Indios». En: Christian F. Feest y Karl-Heinz Kohl (eds.), Hauptwerke der Ethnologie. Stuttgart: Kröner, pp. 1-5.

2003 Die Geschichte der Anderen. Zur Ethnohistorie am Beispiel Perus (15321700). Berlín: Reimer.

2006 «Ethnographie». En: Friedrich Jaeger (ed.). Enzyklopädie der Neuzeit. 16 vols. Stuttgart/Weimar: Verlag J. B. Metzler, pp. 592-594.

2014 «Ethnographie». En: Manfred Landfester (ed.). Renaissance-Humanismus. Lexikon zur Antikerezeption (Der Neue Pauly, Supplemente 9). Stuttgart/Weimar: Verlag J.B. Metzler, pp.358-365.

2017 a «Missionary Reports and their Relevance as Ethnographic Sources». En: Markus Friedrich y Alexander Schunka (eds.), Reporting Christian Missions in the Eighteenth Century: Communication, Culture of Knowledge and Regular Publication in a Cross-Confessional Perspective (Jabloniana 8). Wiesbaden: Harrassowitz Verlag, pp. 19-33.

2017b «Von «grimmigen Menschenfressern» und ¿Edlen Wilden»: Kannibalismus in frühen Reiseberichten aus Amerika zwischen Alteritätsdiskurs und Kulturkritik». En: Robert Rebitsch, Friedrich Pöhl, Sebastian Fink (eds.). Die Konstruktion des Kannibalen zwischen Fiktion und Realität (Philippika 111). Wiesbaden: Harrassowitz Verlag, pp. 127-154.

GemegaH, Helga

1999 Die Theorie des spanischen Jesuiten José de Acosta (ca. 1540-1600) über den Ursprung der indianischen Völker aus Asien (Hispano-Americana 22). Frankfurt am Main et al.: Peter Lang.

Guaman Poma de Ayala, Felipe

1980 El Primer Nueva Corónica y Buen Gobierno [c. 1615]. Ed. crítica de 
John V. Murra y Rolena Adorno. Trad. y análisis textual del quechua por Jorge L. Urioste. 3 vols. México: Siglo Veintiuno Editores.

KONETZKE, Richard

1974 Süd- und Mittelamerika I. Die Indianerkulturen Altamerikas und die spanisch-portugiesische Kolonialherrschaft. (Fischer Weltgeschichte, 22). Frankfurt: Fischer.

LANDFESTER, Manfred

2000 «Der Blick auf das Andere. Herodot und die Anfänge der antiken Berichte über außergriechische Völker und Länder». En: Xenia von Ertzdorff (ed.). Beschreibung der Welt: Zur Poetik der Reise- und Länderberichte (Chloe, Beihefte zum Daphnis, 31). Amsterdam/Atlanta: Rodopi, pp. 3-35.

LÉRY, Jean de

1580 [1578] Histoire d'un voyage faict en la terre du Bresil, autrement dite Amerique. Geneve: Antoine Chuppin.

LESTRINGANT, Frank

1991 André Thevet: Cosmographe des derniers Valois. Genève: Droz.

LÉvi-STrauss, Claude

1955 Tristes tropiques. París: Librairie Plon.

1982 «Eine Idylle bei den Indianern. Über Jean de Léry». En: KarlHeinz Kohl (ed.). Mythen der Neuen Welt. Zur Entdeckungsgeschichte Lateinamerikas. Berlín: Verlag Frölich und Kaufmann, pp. 68-70.

Locke, John

1988 [1689] Two Treatises of Government. Cambridge: Cambridge University Press.

MacCormack, Sabine

1991 Religion in the Andes: Vision and Imagination in Early Colonial Peru. Princeton: Princeton University Press.

MARZAL, Manuel M.

1993 Historia de la antropología indigenista: México y Perú. Barcelona: Anthropos; México: Universidad Autónoma Metropolitana-Iztapalapa. 
O'Gorman, Edmundo

1989 Cuatro historiadores de Indias, siglo XVI. Pedro Mártir de Anglería, Gonzalo Fernández de Oviedo y Valdés, Fray Bartolomé de las Casas, Joseph de Acosta. México, D.F.: Editorial Patria bajo el sello de Alianza Editorial Mexicana [1 $1^{\mathrm{a}}$ ed. 1972].

Plinius SeCundus, Caius

1973 Naturalis Historiae - Naturkunde. Lateinisch - deutsch. Libri XXXVII, Liber 1. Roderich König (ed. y trad.). München: Heimeran Verlag.

TheveT, André

1556 Cosmographie de Levant. Lyon: Ian de Tovrnes et Gvil Gazeav.

1558 Les singularitez de la France antarctique, autrement nommée Amerique, et des plusieurs terres et isles decouvertes de nostre temps. Paris: Chez les heritiers de Maurice de la Porte, au Clos Bruneau, à l'enseigne S. Claude.

VALDEÓn, Roberto A.

2014 Translation and the Spanish Empire in the Americas. Amsterdam/Philadelphia: John Benjamins Publishing Company. 